\title{
Shape Preserving Sampling and Reconstruction of Grayscale Images
}

\author{
Peer Stelldinger \\ Cognitive Systems Group, University of Hamburg, \\ Vogt-Köln-Str. 30, D-22527 Hamburg, Germany
}

\begin{abstract}
The expressiveness of a lot of image analysis algorithms depends on the question whether shape information is preserved during digitization. Most existing approaches to answer this are restricted to binary images and only consider nearest neighbor reconstruction. This paper generalizes this to grayscale images and to several reconstruction methods. It is shown that a certain class of images can be sampled with regular and even irregular grids and reconstructed with different interpolation methods without any change in the topology of the level sets of interest.
\end{abstract}

\section{Introduction}

Much of the information in an analog image may get lost under digitization. An image analysis algorithm can only be successful, if the needed information is preserved during the digitization process. Since a lot of image analysis algorithms are based on level sets, isosurface contours, and their shapes, it is important to know how to guarantee that the shapes of level sets are preserved. Up to now the problem of shape preserving digitization has mostly been dealt with for binary images.

It is well known that so-called $r$-regular binary images (see difinition 1 ) can be digitized with square or hexagonal grids of a certain density without changing the shape in a topological sense $[2,11,12]$. Recently Köthe and the author were able to show that this is true for any grid of a certain density and that this still holds if the image is blurred by a disc shaped point spread function $[7,13]$. In case of square grids, this is also proved for square shaped point spread functions $[8,9]$. But all this work is not only restricted to binary images but also to nearest neighbor reconstruction in combination with thresholding. The only exception is the work of Florêncio and Schafer [2], which allows other morphological reconstruction methods, too, but then only guarantees a bounded Hausdorff error instead of topological equivalence. In general, reconstruction means extending the domain of a discrete image function from the set of sampling points to the whole plane $\mathbb{R}^{2}$. In another paper [3] Florêncio and Schafer show that even certain grayscale images can be sampled and reconstructed with a bounded Hausdorff error, when using a regular grid and some morphological reconstruction method. 
All the mentioned approaches use several different ways to compare an image with its reconstructed digital counterpart. The strongest mentioned similarity criterion is strong $r$-similarity [13], which subsumes the others and which is used in this paper. The prior results are generalized to grayscale images and to a broad class of important reconstruction methods.

\section{Regular Images and 2D Monotony}

In this section some basic concepts are defined, which are necessary for the following work. Namely a definition of $r$-regular graylevel images and a generalization of monotony to $2 \mathrm{D}$ is given. Additionally some connections between these two ideas are shown, which are used in the proofs of the following sections.

At first some basic notations are given: The Complement of a set $A$ will be noted as $A^{c}$. The boundary $\partial A$ is the set of all common accumulation points of $A$ and $A^{c}$. The interior $A^{0}$ of $A$ is defined as $A \backslash \partial A$ and the closure $\bar{A}$ is the union of $A$ and $\partial A$. A set $A$ is open, if $A=A^{0}$ and it is closed if $A=\bar{A}$. $\mathcal{B}_{r}(c):=\left\{x \in \mathbb{R} \mid(x-c)^{2} \leq r^{2}\right\}$ denotes the closed disc and $\mathcal{B}_{r}^{0}(c):=\left(\mathcal{B}_{r}(c)\right)^{0}$ denotes the open disc of radius $r$ and center $c$. The $\varepsilon$-dilation of a set $A$ is defined as the set of all points having a distance of at most $\varepsilon$ to some point in $A . L_{t}(f)$ shall be the level set with threshold value $t$ of an image function $f: \mathbb{R}^{2} \rightarrow \mathbb{R}$ : $L_{t}(f):=\left\{\boldsymbol{x} \in \mathbb{R}^{2} \mid f(x) \geq t\right\}$. A set $A \subset \mathbb{R}^{2}$ is called simple $2 D$ (simple 1D) if it is homeomorphic to the unit disc $\mathcal{B}_{1}(0)$ (to the unit interval). Obviously compact subsets of the plane are simple $2 \mathrm{D}$ iff their boundary is a Jordan curve.

Definition 1. A compact set $A \subset \mathbb{R}^{2}$ is called $r$-regular if for each boundary point of $A$ it is possible to find two osculating open discs of radius $r$, one lying entirely in $A$ and the other lying entirely in $A^{c}$. A grayscale image function $f: \mathbb{R}^{2} \rightarrow \mathbb{R}$ is $r$-regular, if each level set is $r$-regular.

Note, that an $r$-regular grayscale image does not contain isolated extrema or saddle points, but plateaus. Each local extremum is a plateau with $r$-regular shape. The property that extrema become plateaus is similar to the concept of one-dimensional local monotonic functions, as defined in [1]. These functions, which are monotonic in any interval of some restricted size, do not change under median filtering. Additionally they are invariant under morphological opening and closing, which is also true for $r$-regular binary images as already stated by Serra [12]. This suggests to ask for the relationship between the concepts of monotony and $r$-regularity. Therefore a suitable generalization of monotony to $2 \mathrm{D}$ is needed.

Our approach is to understand local monotony as a topological criterion of the neighborhood: When applying an arbitrary threshold function to a 1D locally monotonic function, the resulting binary set can have at most one component in each interval of some restricted size. This can easily be generalized to higher dimensions: 
Definition 2. Let $A \subset \mathbb{R}^{2}$ be a simple 2D set. $A$ closed set $B \subset \mathbb{R}^{2}$ is called monotonic in $A$, if both $B \cap A$ and $\overline{B^{c}} \cap A$ are simple $2 D$, empty or one-pointsets. $B$ is called constant in $\mathrm{A}$ if $B \cap \partial A=\emptyset$. An image function $f: \mathbb{R}^{2} \rightarrow \mathbb{R}$ is called monotonic (constant) in $A$, if $L_{t}(f)$ is monotonic (constant) in $A$ for each threshold value $t \in \mathbb{R}$.

This definition of monotony is a generalization of monotony on paths, since a function is monotonic on some path if the level sets and their complements are simple 1D, empty or one-point-sets. If you have an image function being monotonic in a simple $2 \mathrm{D}$ set $A$, there exists from each point in $A$ a monotonic decreasing path to any minimal boundary point of $A$ and a monotonic increasing path to any maximal boundary point. Figure 1 illustrates, what monotony in 2D means: If an image is monotonic in some area, then this part of the image is homeomorphic to an image which is (in the classical sense) monotonic on each straight line though it.

Definition 3. Let $f: \mathbb{R}^{2} \rightarrow \mathbb{R}$ be an image function and let $A \subset \mathbb{R}^{2}$ be a simple $2 D$ set. Further let $A_{v}=A+\boldsymbol{v}$ be the result of the translation of $A$ by a vector $\boldsymbol{v} \in \mathbb{R}^{2}$. Then $f$ is called locally monotonic w.r.t. $A$, if $f$ is monotonic in $A_{v}$ for any $\boldsymbol{v}$.

Lemma 1. An image function $f$ is locally monotonic w.r.t. a simple $2 D$ set $A$ iff each level set $L_{t}(f)$ with $t \in \mathbb{R}$ is locally monotonic w.r.t. A.

Proof. The lemma follows directly from the definition.

The next lemmas show the connection between local monotony and $r$-regularity. Since 2D monotony is a fundamental property of several reconstruction methods (see section 3), the lemmas explain why $r$-regular images have been used for nearly all shape preserving sampling theorems:

Lemma 2. Let $A$ be a disc with radius smaller than some $r \in \mathbb{R}$ or let $A$ be an intersection of a finite number of such discs. Then every $r$-regular image $f$ is locally monotonic w.r.t. A.

Proof. For each threshold value $t \in \mathbb{R}, L_{t}(f)$ is an $r$-regular set. Due to $r$ regularity, no three boundary points of $L_{t}(f)$ lie on a common circle of some radius smaller than $r$. Since no three boundary points of $A$ lie on a common circle with a radius of at least $r, A$ and $L_{t}(f)$ can have at most two boundary points in common. If $L_{t}(f) \cap A$ or $\overline{\left(L_{t}(f)\right)^{c}} \cap A$ is empty, $L_{t}(f)$ is monotonic in $A$. Otherwise one or two of such boundary points exist, because no component of an $r$-regular image can lie completely in $A$. If there is only one such boundary point, $L_{t}(f) \cap A$ or $\overline{\left(L_{t}(f)\right)^{c}} \cap A$ has to be a one-point-set, which implies monotony. Finally if there are two such boundary points, the boundary part $\partial L_{t}(f) \cap A$ cuts $A$ into two simple 2D parts $L_{t}(f) \cap A$ and $\overline{\left(L_{t}(f)\right)^{c}} \cap A$, which implies monotony. Obviously this is also true for any translated version of $A$ and thus $f$ is locally monotonic w.r.t. $A$. 


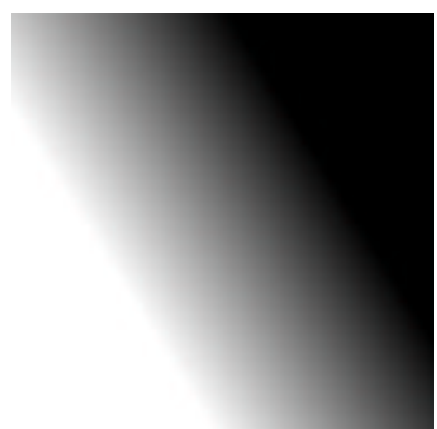

(a)

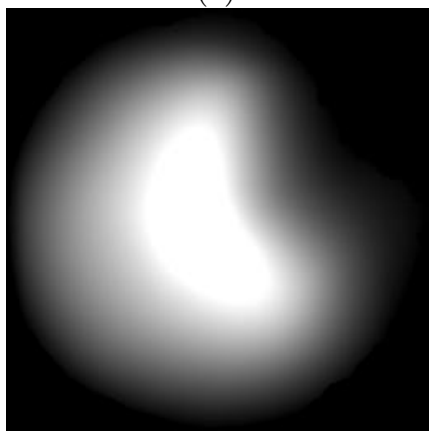

(c)

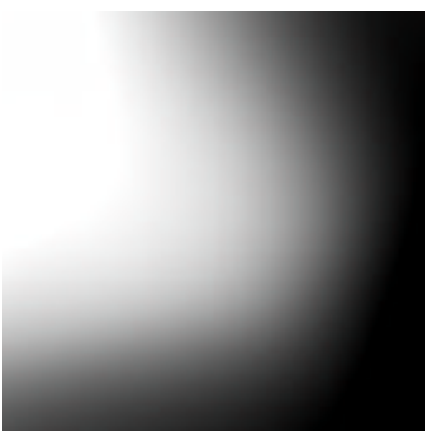

(b)

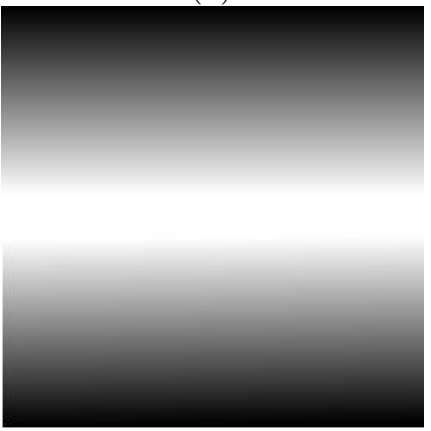

(d)

Fig. 1. The image in (a) is monotonic on each straight line through it. Thus obviously it can be called monotonic in 2D. (b) shows an image, which is homeomorphic to (a), and thus monotonic. There is no local maximum or minimum in the shown area, except of exactly one maximum and one minimum at the boundary. (c) shows a local maximum and thus is not monotonic in the shown area. The same is true for image (d), since there are two minimal regions.

Thus you can take for example any disc shaped area of some $r$-regular image and it will be monotonic, if the disc has some radius smaller than $r$. Or you can take a finite intersection of such areas, e.g. Reuleaux triangles (see [13] for a definition).

Lemma 3. Let $A$ be an $r$-regular set and $B, C \subset \mathbb{R}^{2}$ be two simple $2 D$ sets such that

- $B$ is a subset of some $r^{\prime}$-disc and $C$ is a subset of some $r^{\prime \prime}$-disc with $r^{\prime}, r^{\prime \prime}<r$,

- $A$ is monotonic in both $B$ and $C$,

- $\partial B$ crosses $\partial C$ in exactly two points $p_{1}, p_{2}$,

- $\partial A$ crosses $\partial B$ in exactly two points $b_{1}, b_{2}$ both different from $p_{1}$ and $p_{2}$, and

- $\partial A$ crosses $\partial C$ in exactly two points $c_{1}, c_{2}$ both different from $p_{1}$ and $p_{2}$.

Then $A$ is monotonic either in $\overline{B \backslash C}$ or in $\overline{C \backslash B}$. Furthermore $A$ is monotonic in either $\partial B \cap C$ or $\partial C \cap B$ (see Fig. 2). 


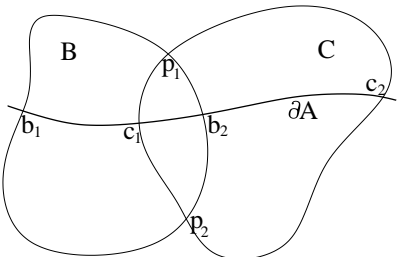

(a)

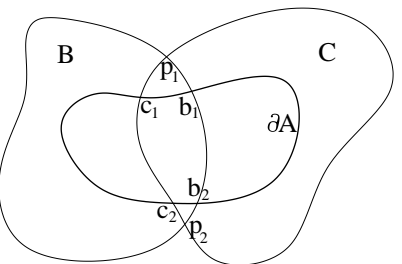

(b)

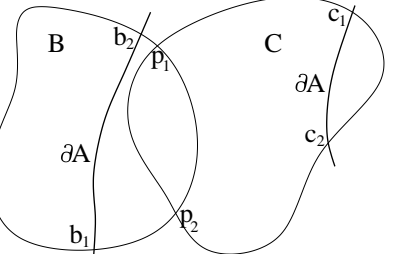

(c)

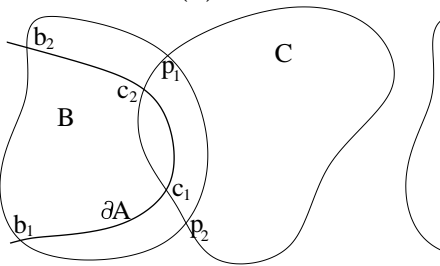

(d)

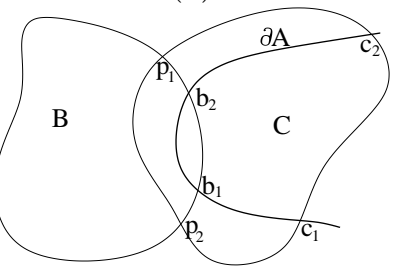

(e)

Fig. 2. There are only 5 possibilities, how $\partial A$ can go through $B$ and $C$, such that their boundaries are each only crossed twice but not in $p_{1}$, and $p_{2}$.

Proof. Figure 2 shows the different possibilities of $\partial A$ going through $B$ and $C$.

Both $\partial A \cap B$ and $\partial A \cap C$ consist of exactly one component, since they cannot be empty and since $A$ is monotonic in $B$ and $C$. Thus $\partial A \cap(B \cup C)$ consists of two components if $\partial A \cap(B \cap C)$ is empty, and of one component otherwise. If $\partial A \cap(B \cap C)$ is empty, $A \cap B \cap C$ is empty or equal to $B \cap C$ (see Fig. 2c) then $A$ is monotonic in both $\overline{B \backslash C}$ and $\overline{C \backslash B}$ and $A$ is even constant in both $\partial B \cap C$ and $\partial C \cap B$.

Otherwise $\partial A \cap(B \cup C)$ must be connected. If the intersection of $A$ and $\overline{B \backslash C}$ is empty or equal to $\overline{B \backslash C}$ (see Fig. 2e) or if the intersection of $A$ and $\overline{C \backslash B}$ is empty or equal to $\overline{C \backslash B}$ (see Fig. 2d), $A$ is monotonic in $\overline{B \backslash C}$ (then $A$ is constant and thus monotonic in $\partial C \cap B$ ) or in $\overline{C \backslash B}$ (then $A$ is constant in $\partial B \cap C)$, respectively.

Else $\partial A \cap(B \cup C)$ must go through $\overline{B \backslash C}, \overline{C \backslash B}$ and $B \cap C$ without meeting $p_{1}$ or $p_{2}$. There are only two remaining possibilities: First, $\partial A$ enters $\overline{B \backslash C}$ at some point $b_{1}$ on $\partial B \backslash C$, next enters $B \cap C$ at some point $c_{1}$ on $\partial B \cap C^{0}$ and leaves it at some point $b_{2}$ on $\partial C \cap B^{0}$, before finally leaving $\overline{C \backslash B}$ through some point $c_{2}$ on $\partial C \backslash B$ (see Fig. 2a). Second, $\partial A$ does not intersect $\partial(B \cup C)$. Starting in $B \backslash C$, it goes through $(B \cap C)^{0}$ into $C \backslash B$ and on another path back through $(B \cap C)^{0}$ into $B \backslash C$ to the starting point (see Fig. 2b). In both cases both $\overline{B \backslash C}$ and $\overline{C \backslash B}$ are cut by $\partial A$ into two simple $2 \mathrm{D}$ parts, which implies monotony. 
In the first case $\partial A$ intersects both $\partial B \cap C$ and $\partial C \cap B$ in only one point and thus $A$ is monotonic on these paths. The second case is in contradiction to the $r$-regularity of $A$, since $B \cup C$ is subset of the union of two discs of radii smaller than $r$ and no such union can cover an $r$-regular set.

Lemma 4. Let $A, B$ be simple 2D sets, such that $A$ is monotonic in $B$ and let $S=\left(s_{0}, s_{1}, \ldots, s_{n}=s_{0}\right)$ be a clockwise ordered cyclic list of points lying on $\partial B$, such that there exist no four points $s_{a}, s_{b}, s_{c}, s_{d}$ with $a<b<$ $c<d$ such that $s_{a}, s_{c} \in A$ and $s_{b}, s_{d} \in A^{c}$ or vice versa. Further let $P=$ $\left\{p_{0,1}, p_{1,2}, \ldots, p_{n-1, n}\right\}, p_{i, j} \subset B$, be a set of non-intersecting (except of their endpoints) simple paths in $B$ between neighboring points of $S$, such that $A$ is monotonic in each path (the existence of such paths is shown in the proof of Theorem 1). Then the area enclosed by the paths is simple $2 D$ and $A$ is monotonic in it.

Proof. The paths define a closed curve. Since they do not intersect (except of their endpoints), this curve is a jordan curve. The closed set $C$, which is circumscribed by this curve, is a simple $2 \mathrm{D}$ set and a subset of $B$. Each path $p_{i}$ cuts $\partial A \cap B$ in at most two parts, where only one part can intersect $C$. It follows by induction that $\partial A \cap C$ consists of at most one component, hitting $\partial C$ in two points. This component separates $C$ into two simple $2 \mathrm{D}$ sets (bounded by jordan curves) $A \cap C$ and $\overline{A^{c}} \cap C$. Thus $A$ is monotonic in $C$.

\section{Sampling and Reconstruction}

In order to compare analog with digital images, a definition of the processes of sampling and reconstruction is needed. The most obvious approach for sampling is to restrict the domain of the image function to a set of sampling points. This set is called a sampling grid. In most approaches only special grids like square or hexagonal ones are taken into account $[2,3,4,5,8,9,11,12]$. A more general approach only needs that a grid is a countable subset of $\mathbb{R}^{2}$, with the sampling points being not too sparse or too dense anywhere $[6,7,13]$. There the pixel shapes are introduced as Voronoi regions.

Definition 4. A countable set $S \subset \mathbb{R}^{2}$ of sampling points, where the Euclidean distance from each point $\boldsymbol{x} \in \mathbb{R}^{2}$ to the next sampling point is at most $r \in \mathbb{R}$, is called an $r$-grid if for each bounded set $A \in \mathbb{R}^{2}$ the subset $S \cap A$ is finite. The Voronoi region of a sampling point is the set of all points lying at least as near to this point than to any other sampling point. A maximal set of sampling points $s_{i}$, whose Voronoi regions have a common corner point $p$, is called Delaunay tuple and its convex hull is the Delaunay cell. Obviously, the sampling points $s_{i}$ lie on a common circle with center $p$ (see Fig. 3c). $p$ is called the center point of the Delaunay cell. An r-grid is named degenerated if at least one of the associated Delaunay cells (not necessarily triangles) does not contain its center point. A Delaunay tuple is called well composed regarding to an image function $f$, if the clockwise ordered cyclic list of sampling points $s_{1}, s_{2}, \ldots s_{n-1}, s_{n}=s_{0}$ has only 


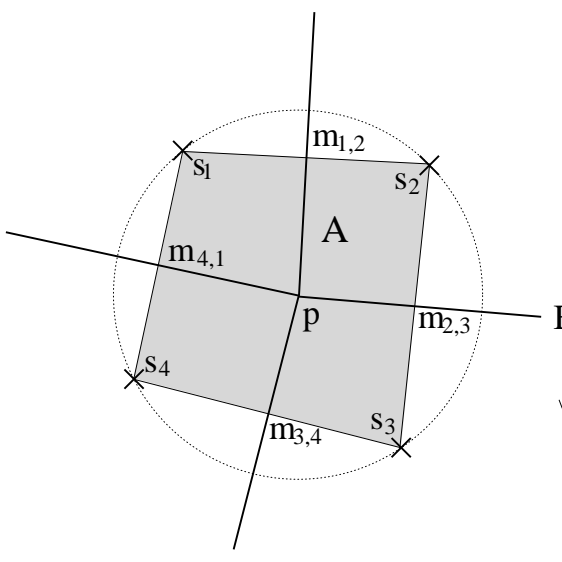

(a)

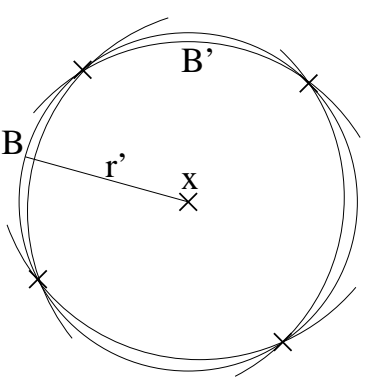

(b)

Fig. 3. (a): Definition of linear reconstruction (definition 5). (b): construction of monotonic covering of a Delaunay cell (Theorem 1).

one local maximum and one local minimum (plateaus are allowed) in $f$, or in other words that there exist no four points $s_{a}, s_{b}, s_{c}, s_{d}$ with $a<b<c<d$ such that

$f\left(s_{a}\right)>f\left(s_{b}\right), f\left(s_{b}\right)<f\left(s_{c}\right), f\left(s_{c}\right)>f\left(s_{d}\right)$ and $f\left(s_{d}\right)<f\left(s_{a}\right)$, or $f\left(s_{a}\right)<f\left(s_{b}\right), f\left(s_{b}\right)>f\left(s_{c}\right), f\left(s_{c}\right)<f\left(s_{d}\right)$ and $f\left(s_{d}\right)>f\left(s_{a}\right)$.

Note, that for any well composed set in the sense of Latecki [9], each Delaunay tuple is well composed. The definition even matches the definition of extended well composed sets by Wang and Bhattacharya [14].

Since reconstruction is a local process where the value of a point is influenced by the surrounding sampling points, several reconstruction methods are defined piecewise. One obvious idea is to define the reconstruction for each Delaunay cell of the sampling grid separately. Then the basic idea is that an image, which is locally monotonic, should have a locally monotonic reconstruction. Therefore a class of such reconstruction functions is defined, which includes generalizations of bilinear and nearest neighbor reconstruction to arbitrary grids.

Definition 5. Let Img be the class of all grayscale image functions $f: \mathbb{R}^{2} \rightarrow \mathbb{R}$ and let $S \subset \mathbb{R}^{2}$ be an r-grid for some $r$. A function $\operatorname{rec}_{S}: \operatorname{Img} \rightarrow \operatorname{Img}$ is called a reconstruction function if $\forall f \in \operatorname{Img}, \forall s \in S:\left(\operatorname{rec}_{S}(f)\right)(s)=f(s)$ and $\forall f, g \in \operatorname{Img}:\left.f\right|_{S}=\left.g\right|_{S} \Rightarrow \operatorname{rec}_{S}(f)=\operatorname{rec}_{S}(g)$. A reconstruction function is called Delaunay-monotonic if for each well composed Delaunay tupel the reconstruction is monotonic in the corresponding Delaunay cell, and - restricted to this cell each nonempty level set of the reconstruction and its complement contains at least one sampling point, respectively. 
Now let $S$ be a non-degenerated grid and $f$ be an image function.

The linear reconstruction function $\operatorname{rec}_{S}$ is defined as follows. Let $A$ be a Delaunay cell of the grid and p be its center point (see Fig. 3a). Then the Delaunay cell can be divided by the median lines from $p$ to the boundary edges of the Delaunay cell into quadrangles. Now the reconstruction function is defined by a bilinear interpolation in each of the quadrangles, where the values for the four vertices are the following: At the sampling points $s_{i}, \operatorname{rec}_{S}(f)$ is equal to $f$; at $p$, $\operatorname{rec}_{S}(f)$ is the mean of the values of $f$ at the sampling points ( $p$ has equal distance to all of these sampling points); at the median points $m_{i, j}$, $\operatorname{rec}_{S}(f)$ is the mean value of the two corresponding sampling points $s_{i}, s_{j}$.

The nearest neighbor reconstruction function $\operatorname{rec}_{S}$ is defined by giving each point the value of the nearest of the sampling points which correspond to the actual Delaunay cell. If there is no unique nearest sampling point, the one with the highest value is taken.

In a Delaunay-monotonic reconstruction, the image is monotonic in each Delaunay cell, where this is possible. Of course it cannot be monotonic in a Delaunay cell where the clockwise ordered cyclic list of sampling points has not only one local maximum and one local minimum in the image function.

In case of square grids, these definitions are equivalent to the standard bilinear interpolation and the nearest neighbor interpolation. Any linear or nearest neighbor reconstruction function is Delaunay-monotonic, since no overshooting can occur. Note that even more complex reconstruction methods like biquadratic interpolation only need slight modifications (cutting off the overshootings) in order to be Delaunay-monotonic. In case of the nearest neighbor reconstruction, the value of each sampling point is simply set to the whole Voronoi region, which is equal to the reconstructions used in $[2,3,7,8,9,11,12,13]$. Even the marching squares algorithm, a two-dimensional simplification of the well-known marching cubes algorithm [10], defines a Delaunay-monotonic reconstruction function.

\section{Shape Preserving Sampling Theorems}

In this section at first two minor sampling theorems are proved. The first theorem is only for binary images, where no sampling point lies on the boundary of any foreground component. The second theorem extends this to any binary image. After that the third and main result finally generalizes these theorems to grayscale images.

In the following, the well-defined similarity criterion strong $r$-similarity (see [13]) is used to compare shapes before and after digitization. Two shapes $A, B \subset$ $\mathbb{R}^{2}$ being strongly $r$-similar means that there exists a homeomorphic deformation $f$ of the plane $\mathbb{R}^{2}$, with $f(A)=B$ and where the movement of each point is 
bounded by $r: \forall \boldsymbol{x} \in A:|\boldsymbol{x}-f(\boldsymbol{x})| \leq r$. Such a restricted homeomorphism is called $r$-homeomorphism. This criterion is stricter than both the preservation of topology used by Pavlidis and Latecki et al. [8,9,11] and the isomorphy of homotopy trees used by Serra [12]. It additionally sets a bound for the Hausdorffdistance of the original and the reconstructed set and of their boundaries. Strong $r$-similarity is originally a criterion for binary images like shapes. If we have a grayscale image, we can investigate the shapes given by the level sets of the image. So two grayscale images are called strongly $r$-similar if this is true for all of their level sets.

Theorem 1. Let $A$ be an $r$-regular set and $S$ be a non-degenerated $r^{\prime}$-grid with $r^{\prime}<r$, such that no sampling point $x \in S$ lies on $\partial A$. Further let $\operatorname{rec}_{S}$ be a Delaunay-monotonic reconstruction function. Then there exists a $2 r^{\prime}$-homeomorphism from $A$ to the reconstruction $L_{v}\left(\operatorname{rec}_{\mathcal{S}}(A)\right)$ for each threshold value $v \in(0,1)$.

Proof. In the following such a homeomorphism is defined by partitioning the original image and the reconstruction into homeomorphic parts corresponding to the Delaunay cells.

Let $p$ be the center point of some Delaunay cell of the grid. Then there exists a disc with radius of at most $r^{\prime}$ and center in $p$, such that each element of the Delaunay tuple lies on its boundary. The set $X$ of all so defined discs (one for each corner point) covers the whole plane and $A$ is monotonic in each disc.

Now we replace each disc $B$ by the intersection $B^{\prime}$ of new discs of slightly bigger radius $r^{\prime \prime}<r$, which cover all the sampling points lying on $\partial B$ and which each have exactly two neighboring sampling points on their boundary (see Fig. $3 \mathrm{~b})$. Doing this with an appropriate radius $r^{\prime \prime}$ we can guarantee that $\partial A \cap B^{\prime}$ is no one-point-set. The so constructed set $X^{\prime}$ still covers the plane, while $A$ being monotonic in each element of $X^{\prime}$ according to Lemma 2.

Due to the construction of $X^{\prime}$, the boundaries of each two elements of $X^{\prime}$ intersect in two or zero points. Now let $B_{1}, B_{2}$ be two elements of $X^{\prime}$, such that their boundaries intersect in two points $p_{1}$ and $p_{2}$. Since the interior of each element of $X^{\prime}$ does not contain any sampling point, the path $\partial B_{1} \cap B_{2}$, going from $p_{1}$ to $p_{2}$ does not hit any sampling point except of the possible sampling points $p_{1}$ and $p_{2}$. If $p_{1}$ and $p_{2}$ are both sampling points, we can choose one such path $P$ between them due to Lemma 3, with $A$ being monotonic in $P$. By doing this for every pair of neighboring sampling points we can map each edge of the Delaunay graph to such a path. Each of these paths is covered by both discs of $X$, which intersect with the endpoints of the path. Now we modify this set of paths $Y$, such that no two paths intersect in non-sampling points:

If two paths intersect in a common subpath, which is not an isolated point, we can displace one path in this area by a small distance, such that the two 

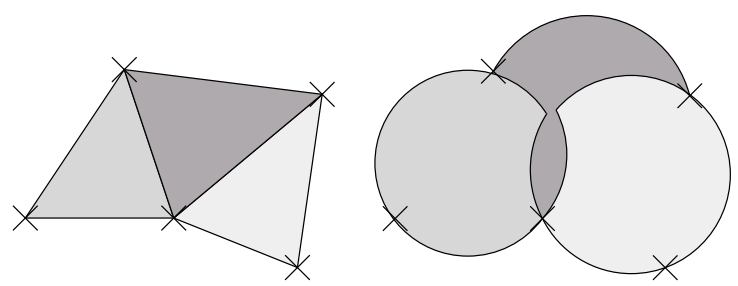

Fig. 4. The reconstructed image function is monotonic in each Delaunay cell (colored regions in the left figure). One can find corresponding regions in the original image, where the image function is monotonic, too (colored regions in the right figure).

new paths intersect only in the endpoints of the formerly common subpath and in common intersection points with $\partial A$. This is possible without changing the monotony of $A$ on the paths since $\partial A$ intersects the paths in at most one isolated point. The resulting paths can be guaranteed to be covered by the corresponding elements of $X$, due to the construction of $X^{\prime}$. Thus the resulting paths only intersect in isolated points. Now let $P_{1}$ and $P_{2}$ be two paths, crossing each other in two points $p_{1}$ and $p_{2}$. Then by swapping the parts of the paths between $p_{1}$ and $p_{2}$ we get two paths, which intersect in $p_{1}$ and $p_{2}$, but do not cross each other in these points. If two paths cross each other in only one point there are two cases: First, if they intersect also in an endpoint we can use the same swapping technique. Second, if they intersect in no other point, one of the endpoints of one path must be enclosed by the circular set of paths covered by one of the corresponding discs of $X$, which is impossible since no sampling point lies in the interior of such a disc.

Since the swapped path parts are covered by the same discs of $X$, the covering properties do not change under this swap operation. By induction we get a set of paths $Y^{\prime}$ where no two paths cross each other. The paths can only intersect at some single points without crossing. At these points we can displace one path by a small distance such that the paths do not intersect anymore.

All these path modifications do not change the monotony of $A$ on them. Thus the resulting set of paths defines an embedding of the Delaunay graph into the plane, such that each region can be covered by an $r^{\prime}$-disc. Figure 4 shows an example of neighboring Delaunay cells and their corresponding embeddings in the reconstructed image. With Lemma 4 the Delaunay cells and their corresponding regions due to the above construction are each homeomorphic. Since two such corresponding regions can be covered by a common $r^{\prime}$-disc, each homeomorphism is a $2 r^{\prime}$-homeomorphism. Obviously we can choose homeomorphisms which are identical on the constructed paths, which implies strong $r^{\prime}$-similarity of $A$ and $L_{v}\left(\operatorname{rec}_{\mathcal{S}}(A)\right)$ for each theshold value $v \in(0,1)$. 
This means that if a shape is $r$-regular, it can be sampled with any sampling grid of a certain density and any Delaunay-monotonic reconstruction method, such that the resulting reconstruction has exactly the same topological properties and only a bounded Hausdorff distance to the original shape. The only restriction is that the sampling points are not allowed to lie on the shape boundary. The next theorem shows that this restriction is not really existent.

Theorem 2. Let $A$ be an $r$-regular set and $S$ be a non-degenerated $r^{\prime}$-grid with $r^{\prime}<r$. Further let rec be a Delaunay-monotonic reconstruction function. Then there exists for any $\varepsilon>\mathbb{R} a\left(2 r^{\prime}+\varepsilon\right)$-homeomorphism from $A$ to the reconstruction $L_{v}\left(\operatorname{rec}_{\mathcal{S}}(A)\right)$ for each threshold value $v \in(0,1)$.

Proof. Let $d$ be the minimal distance between $A$ and any sampling point not lying in $A$ - this is uniquely defined since there is only a finite number of sampling points having a distance of at most $r$ due to the compactness of $A$. Now let $\varepsilon$ be any number with $0<\varepsilon<\min \left(d, r-r^{\prime}\right)$. Then the $\varepsilon$-dilation $A^{\prime}$ of $A$ is $\varepsilon$-homeomorphic to $A$ and has exactly the same reconstruction, since the values at the sampling points did not change. Due to Theorem 1 the $(r-\varepsilon)$ regular set $A^{\prime}$ is $\left(2 r^{\prime}\right)$-homeomorphic to the reconstruction and in case of the nearest neighbor reconstruction even $r^{\prime}$-homeomorphic to it. The concatenation of the two homeomorphisms defines a $\left(2 r^{\prime}+\varepsilon\right)$-homeomorphism from $A$ to the reconstruction $L_{v}\left(\operatorname{rec}_{\mathcal{S}}(A)\right)$.

In case of nearest neighbor reconstruction the similarity bound is even stronger. In [13] Köthe and the author showed that then even strong $r^{\prime}$-similarity instead of strong $\left(2 r^{\prime}\right)$-similarity is given.

Now the final step to grayscale images is straightforward, since each level set of an $r$-regular grayscale image is $r$-regular, too.

Corollary 1. Let $f$ be an r-regular image function and $S$ be a non-degenerated $r^{\prime}$-grid with $r^{\prime}<r$. Further let rec be a Delaunay-monotonic reconstruction function. Then the reconstruction is strongly $(2 r)$-similar to $f$ for any theshold value, which is not equal to the image value at some sampling point.

If the threshold value is equal to the image value at some sampling point, the corresponding level set of the reconstructed image function is not necessarily simple 2D, but can contain one dimensional parts and thus the topology changes. But one can show that this does not happen, if the original image is $2 r$-regular, because then each plateau is reconstructed topologically correctly.

\section{Conclusions}

It was proved that any grayscale image, which has only $r$-regular level sets, can be sampled by arbitrary sampling grids of sufficient density and reconstructed by a non-overshooting interpolation method, and still remains strongly $r^{\prime}$-similar (for some bounded $r^{\prime}$ ) for any threshold value, which is not an image value at 
some sampling point. This implies that most level sets do not change topology under digitization and thus you can say the topology of the image is preserved. Each maximum or minimum plateau of the original image can be found in the digitization having the same height.

\section{References}

1. Acton, S.T.: A PDE technique for generating locally monotonic images. Proceedings of the IEEE International Conference on Image Processing, 1998.

2. Florêncio, D.A.F., Schafer, R.W.: Homotopy and Critical Morphological Sampling. Proceedings of SPIE Visual Communications and Image Processing, pp. 97-109, 1995.

3. Florêncio, D.A.F., Schafer, R.W.: Critical Morphological Sampling and its Applications to Image Coding. In J. Serra, P. Soille (Eds.): Mathematical Morphology and Its Applications to Image Processing, Computational Imaging and Vision, vol. 2, pp. 109-116, Kluwer Academic Publishers, Dordrecht, 1994.

4. Haralick, R.M., Zhuang, X., Lim, C., Lee, J.S.J.: The Digital Morphological Sampling Theorem. IEEE Transactions on Acoustics, Speech and Signal Processing 37, pp. 2067-2090, 1989.

5. Heijmans, H.J.A.M., Toet, A.: Morphological Sampling. Computer Vision, Graphics and Image Processing: Image Understanding 54, pp. 384-400, 1991.

6. Herman, G.T.: Geometry of Digital Spaces. Birkhäuser Boston, 1998.

7. Köthe, U., Stelldinger, P.: Shape Preserving Digitization of Ideal and Blurred Binary Shapes. In: I. Nyström et al. (Eds.): DGCI 2003, LNCS 2886, pp. 82-91, Springer, 2003.

8. Latecki, L.J., Conrad, C., Gross, A.: Preserving Topology by a Digitization Process. Journal of Mathematical Imaging and Vision 8, pp. 131-159, 1998.

9. Latecki, L.J.: Discrete Representation of Spatial Objects in Computer Vision. Kluwer, 1998.

10. Lorensen, W.E., Cline, H.E.: Marching Cubes: A High Resolution 3D Surface Construction Algorithm. Computer Graphics 21, no. 4, 1987.

11. Pavlidis, T.: Algorithms for Graphics and Image Processing. Computer Science Press, 1982.

12. Serra, J.: Image Analysis and Mathematical Morphology. Academic Press, 1982.

13. Stelldinger, P., Köthe U.: Shape Preservation During Digitization: Tight Bounds Based on the Morphing Distance. In: B. Michaelis, G. Krel (Eds.): Pattern Recognition, LNCS 2781, pp. 108-115, Springer, 2003.

14. Wang, Y., Bhattacharya, P.: Digital Connectivity and Extended Well-Composed Sets for Gray Images. Computer Vision and Image Understanding 3, pp. 330 345,1997 . 